plification after cessation of treatment with a drug that has a long half-life $(5.5$ months for bedaquiline). ${ }^{5}$

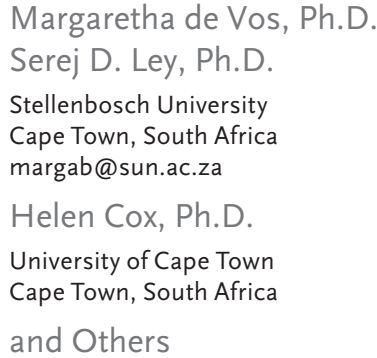

Drs. de Vos and Ley and Drs. Warren and Cox contributed equally to this letter.

A complete list of authors is available with the full text of this letter at NEJM.org.

Use of trade names is for identification only and does not constitute endorsement by the Department of Health and Human Services, the Public Health Service, or the Centers for Disease Control and Prevention (CDC). The findings and conclusions in this letter are those of the authors and do not necessarily represent the views of the CDC, the National Institutes of Health (NIH), or the South African Medical Research Council.

This is the New England Journal of Medicine version of record, which includes all Journal editing and enhancements. The Author Final Manuscript, which is the author's version after external peer review and before publication in the Journal, is available under a CC BY license at PMC6518951.

Supported by the National Research Foundation, the South African Medical Research Council, the Stellenbosch University
Faculty of Medicine Health Sciences, and a grant from the NIH (R01AI131939). Dr. Cox is supported by a Wellcome Trust fellowship (099818/Z/12/Z); Drs. de Vos, Ley, van Rie, and Warren are supported by TORCH funding through the Flemish Fund for Scientific Research (FWO G0F8316N); Dr. Ley is supported by a grant from the Swiss National Science Foundation (P2BSP3 _165379); and Dr. Theron is supported by the EDCTP2 program supported by the European Union (grant SF1401, OPTIMAL DIAGNOSIS). The sequencing work was performed at the Translational Genomics Research Institute and supported by a grant from the Bill and Melinda Gates Foundation (OPP1115887, to Dr. Schito) for the ReSeqTB sequencing platform.

Disclosure forms provided by the authors are available with the full text of this letter at NEJM.org.

1. Schnippel K, Ndjeka N, Maartens G, et al. Effect of bedaquiline on mortality in South African patients with drug-resistant tuberculosis: a retrospective cohort study. Lancet Respir Med 2018;6:699-706.

2. Conradie F, Meintjes G, Hughes J, et al. Clinical Access to Bedaquiline Programme for the treatment of drug-resistant tuberculosis. S Afr Med J 2014;104:164-6.

3. Colman RE, Anderson J, Lemmer D, et al. Rapid drug susceptibility testing of drug-resistant Mycobacterium tuberculosis isolates directly from clinical samples by use of amplicon sequencing: a proof-of-concept study. J Clin Microbiol 2016;54: 2058-67.

4. Andries K, Villellas C, Coeck N, et al. Acquired resistance of Mycobacterium tuberculosis to bedaquiline. PLoS One 2014; 9(7):e102135.

5. McLeay SC, Vis P, van Heeswijk RP, Green B. Population pharmacokinetics of bedaquiline (TMC207), a novel antituberculosis drug. Antimicrob Agents Chemother 2014;58:5315-24.

DOI: 10.1056/NEJMc1815121

\title{
Preventing Venous Thromboembolism in Patients with Cancer
}

TO THE EDITOR: Central venous catheters are commonly used for long periods in patients with cancer, and a nonnegligible increased risk of venous thromboembolism is associated with these devices. ${ }^{1}$ Nonetheless, the presence of a central venous catheter is not included as a component of established risk scores to determine which patients could benefit from thromboprophylaxis. We and others have reported on the role of anticoagulation (heparin or vitamin $\mathrm{K}$ antagonists) in reducing the risk of venous thromboembolism related to the use of a central venous catheter. ${ }^{1,2}$ Carrier et al. (Feb. 21 issue) ${ }^{3}$ report the results of the Apixaban for the Prevention of Venous Thromboembolism in High-Risk Ambulatory Cancer Patients (AVERT) trial, and, in the same issue of the Journal, Khorana et al. ${ }^{4}$ report the results of the CASSINI trial. These two pivotal trials evaluated factor Xa inhibitors for the prevention of venous thromboembolism in patients with cancer. The accompanying editorial by Agnelli ${ }^{5}$ elegantly merged the data from these trials, which showed a reduced risk of venous thromboembo- lism among patients who received thromboprophylaxis with factor Xa inhibitors.

Unfortunately, neither trial stratified patients or reported trial outcomes with adjustment for central venous catheter-related venous thromboembolism. Therefore, we strongly encourage researchers from both trial groups to explore the effect of central venous catheters. Although this was not a prespecified analysis, it would increase our knowledge of the roles of central venous catheters and of factor Xa inhibitors as independent risk factors for venous thromboembolism. This information would help us to evaluate whether the use of central venous catheters should be considered in the decision-making process regarding thromboprophylaxis.

\footnotetext{
Lorenzo D’Ambrosio, M.D., Ph.D.

Massimo Aglietta, M.D.

Giovanni Grignani, M.D.

Candiolo Cancer Institute IRCCS

Candiolo, Italy

lorenzo.dambrosio.md@gmail.com
} 
No potential conflict of interest relevant to this letter was reported.

1. D'Ambrosio L, Aglietta M, Grignani G. Anticoagulation for central venous catheters in patients with cancer. N Engl J Med 2014;371:1362-3.

2. Kahale LA, Tsolakian IG, Hakoum MB, et al. Anticoagulation for people with cancer and central venous catheters. Cochrane Database Syst Rev 2018;6:CD006468.

3. Carrier M, Abou-Nassar K, Mallick R, et al. Apixaban to prevent venous thromboembolism in patients with cancer. $\mathrm{N}$ Engl J Med 2019;380:711-9.

4. Khorana AA, Soff GA, Kakkar AK, et al. Rivaroxaban for thromboprophylaxis in high-risk ambulatory patients with cancer. N Engl J Med 2019;380:720-8.

5. Agnelli G. Direct oral anticoagulants for thromboprophylaxis in ambulatory patients with cancer. N Engl J Med 2019;380:781-3.

DOI: 10.1056/NEJMc1904003

DR. CARRIER AND COLLEAGUES REPLY: Stratification of ambulatory patients with cancer according to their underlying risk of venous thromboembolism is challenging. As highlighted by D'Ambrosio et al., known important risk factors may not be included in risk scores such as the Khorana score (range, 0 to 6 , with higher scores indicating a higher risk of venous thromboembolism). For example, although central venous catheters may be associated with an increased risk of venous thromboembolism, 223 of the 574 patients (38.9\%) who underwent randomization in the AVERT trial had a central venous catheter in place, and a proximal deep-vein thrombosis of the upper limbs developed in only 7 of these 223 patients (3.1\%) over a period of 6 months.

The role of direct oral anticoagulants in preventing deep-vein thrombosis of the upper limbs is the subject of an ongoing pilot randomized trial assessing feasibility (ClinicalTrials.gov number, NCT03506815). ${ }^{1}$ Nonetheless, the Khorana score appears to be clinically relevant. Although approximately two thirds of the patients enrolled in our trial had a Khorana score of 2, the risk of a major venous thromboembolism was high in this subgroup: it occurred in 16 of 190 patients in the placebo group (8.4\%) and in 6 of 186 patients in the apixaban group (3.2\%) (risk difference, -5.2 percentage points; $95 \%$ confidence interval, -10.3 to -3.7$)$.

Marc Carrier, M.D.

Gregoire Le Gal, M.D.

Philip S. Wells, M.D.

Ottawa Hospital Research Institute

Ottawa, ON, Canada

mcarrier@toh.ca

Since publication of their article, the authors report no further potential conflict of interest.
1. Ikesaka R, Langlois N, Carrier M, Kearon C, Le Gal G. Pilot trials in thrombosis: purpose and pitfalls. Res Pract Thromb Haemost 2018;2:572-9.

DOI: 10.1056/NEJMc1904003

DR. KHORANA AND COLLEAGUES REPLY: D'Ambrosio and colleagues raise an important question regarding the risk of thrombosis in an upper limb among patients with central venous catheters. Prophylactic anticoagulation is not well established because of conflicting data and lower rates of thromboembolism reported in contemporary studies ${ }^{1}$ than in previous studies. ${ }^{2} \mathrm{~A}$ Khorana score of 3 or higher has been shown to be a risk factor for such events. ${ }^{3}$ In the CASSINI trial, which involved patients with a Khorana score of 2 or higher, 424 of 841 patients had a central venous catheter. Of these, 1 of 218 patients ( $0.5 \%)$ in the rivaroxaban group and 5 of 206 patients (2.4\%) in the placebo group were adjudicated to have a deep-vein thrombosis in an upper limb while receiving the trial drug or placebo. In addition, excluding infectious complications, $2.8 \%$ of the patients in the rivaroxaban group and $4.9 \%$ in the placebo group had a catheter failure or other complications of a central venous catheter during the trial period. Our exploratory post hoc analysis suggests a reduced risk of thrombosis in an upper limb and noninfectious catheter failure with the use of daily rivaroxaban; these findings may be helpful in decision making regarding the use of thromboprophylaxis in outpatients with cancer.

Alok A. Khorana, M.D.

Cleveland Clinic

Cleveland, $\mathrm{OH}$

khorana@ccf.org

Jodi Mones, M.D.

Gerald A. Soff, M.D.

Memorial Sloan Kettering Cancer Center

New York, NY

Dr. Mones reports receiving research support from Janssen to her institution. Since publication of his article, Dr. Soff reports receiving consulting fees from Bristol-Myers Squibb and Pfizer. No further potential conflict of interest relevant to this letter was reported.

1. Kahale LA, Tsolakian IG, Hakoum MB, et al. Anticoagulation for people with cancer and central venous catheters. Cochrane Database Syst Rev 2018;6:CD006468.

2. Prandoni P. Prophylaxis of catheter-related thrombosis in cancer patients. Lancet 2009;373:523-4.

3. Hohl Moinat C, Périard D, Grueber A, et al. Predictors of venous thromboembolic events associated with central venous port insertion in cancer patients. J Oncol 2014;2014:743181.

DOI: 10.1056/NEJMc1904003 